many of these patients may also be taking high amounts of potassium. The problem is compounded by the fact that potassium overloading may go unnoticed for a long time as hyperkalaemia has few or only vague clinical warning symptoms, and serum potassium concentrations may rise to dangerous concentrations before presentation, as happened in this patient. This event in our patient was almost fatal, and it could readily recur unless a regular check is kept on prescribed drugs, as well as over the counter medicines and products from health shops.

Doctors should thus be careful about their advice on low salt diets, especially for those high risk subjects with underlying cardiac, hepatic, or renal insufficiency, those taking non-steroidal anti-inflammatory drugs, and elderly people. Manufacturers should also clearly state the risks of their preparations in susceptible subjects: the warning should appear prominently on the label.
We thank Mrs Yvonne Bradburn, dietitian and Mrs Joan Kay for typing the manuscript.

Funding: None.

Conflict of interest: None.

1 Hyneck ML. An overview of the clinical pharmacokinetics of nabumetone. I Rheumatol 1992;19(Suppl 36):20-4.

2 Boers M. Renal disorders in rheumatoid arthritis. Semin Arthritis Rheum 1990;20:57-68.

3 Segasothy M, Chin GL, Sia KK, Zulfiqar, Samad SA. Chronic nephrotoxicity of anti-inflammatory drugs used in the treatment of arthritis. $\mathrm{Br} \mathcal{F}$ Rheumatol 1995;34:162-5.

4 Whelton A. Non-steroidal anti-inflammatory drugs: effects on kidney function. F Clin Pharmacol 1991;31:588-98.

5 Stillman MT, Naper J, Blackshear JL. Adverse effects of non-steroidal antiinflammatory drugs on the kidney. Med Clin North Am 1984;68:371-85.

6 Pal B, Hussain MA. Non-steroidal anti-inflammatory drugs and adverse rena effects. Ann Rheum Dis 1985;44:212-3.

7 Reeves WB, Foley RJ, Weinman EJ. Renal dysfunction from non-steroidal anti-inflammatory drugs. Arch Intern Med 1984;144:1943-4.

8 Swales JD. Salt substitutes and potassium intake [editorial]. BMf 1991;303: 1084-5.

(Accepted 1 May 1995)

\title{
Guide to the Internet
}

\section{Electronic mail}

\section{Mark Pallen}

\section{Internet}

This is the second in a short series introducing medical practitioners to the Internet

\section{Department of Medical Microbiology, St Bartholomew's Hospital Medical College, London EC1A 7BE Mark Pallen, senior lecturer}

Correspondence to: m.pallen@ic.ac.uk
Electronic mail (email) has many advantages over other forms of communication: it is easy to use, free of charge, fast, and delivers information in a digital format. As a text only medium, email is usually less formal in style than conventional correspondence and may contain acronyms and other features, such as smileys, that are peculiar to the Internet. Email client programs that run on your own microcomputer render email powerful and easy to use. With suitable encoding methods, email can be used to send any kind of computer file, including pictures, sounds, programs, and movies. Numerous biomedical electronic mailing lists and other Internet services are accessible by email.

Electronic mail, or email, is the transmission of text based messages between networked computers. Email was one of the earliest and most basic resources on the Internet and in many ways it still acts as the lowest common denominator for computer communications. Many computers that cannot access other Internet services can still exchange email with machines on the Internet.

\section{Why use email?}

Several studies have stressed the beneficial effects of communication by email in medical, nursing, and other settings. ${ }^{1-5}$ Indeed, email has many advantages over other methods of communication:

- It is faster than conventional mail (or "snailmail"), usually reaching its destination in minutes, even when sent from the United Kingdom to North America or Australia

- It requires less physical effort than sending a letter by snailmail, as there is no need to buy stamps or envelopes or for a trip to the postbox

- Once the hardware, software, and Internet connection are in place, email on the Internet is free, even if messages are sent to the other side of the world

- Unlike communication by telephone, email does not require the attention of both parties at the same timeso doctors and patients may find it more suitable than the telephone for communication about simple and non-urgent problems ${ }^{67}$

- With email it is easy to send the same piece of information to several people simultaneously-for example, you can circulate memos, agendas, and minutes, or disseminate educational material ${ }^{8}$

- Junk email is rare, so reading the day's email is usually far more pleasurable than sifting through a pile of snailmail; furthermore, you can scan the headers of any new batch of email to separate the urgent from the less important

- If you use an encryption system such as Pretty Good Privacy (PGP) you can render your email messages completely uncrackable to outsiders but easily readable by your intended recipient (however, note that such strong encryption is illegal in some countries and that it is illegal to export PGP from the United States)

- Email delivers the information in a computer readable format - this means that if you are collaborating on, say, a scientific paper, you can easily exchange amended drafts of the paper many times with your coauthor without either of you having to type in the other's amendments.

\section{Essentials of an email message}

An email message consists of a header and a body (fig 1). The header is a kind of virtual envelope, containing some or all of the following lines:

- A "From:" line showing who the message is from - A "Date:" line showing when it was sent

- A "To:" line showing the email address(es) of the intended recipient(s) (each separated by a comma and a space, if more than one)

- A "Subject:" line, which contains a description of the subject matter of the message (leaving the subject line blank is a sure sign of a "newbie"). Most email programs automatically preface the subject line of a follow up or forwarded message with "Re:"

- A "CC:" line showing those who will receive the message in addition to the addressee(s)

- A "Message-ID:" line showing the number 
assigned to the message by the mail program at the host machine

- A series of "Received:" lines showing details of the systems through which the email has passed (useful for troubleshooting if mail bounces back)

- A "Reply-to:" line giving the preferred address for replies (usually but not always the same as the sender's address).

Internet email addresses are written in a standard format, where the username comes first, followed by an @ sign (pronounced "at") and then the user's location, for example

mjp123@bccmsa.bc.ic.ac.uk

No blank spaces are allowed in an email address, nor does it matter whether capital or small letters are used. The location can be broken down into a series of geographical or organisational domain codes, each separated by a "." (pronounced "dot"). Working from right to left, these tell each of the computers along the route between sender and recipient (the "routers") where next to send the email message. The rightmost, or highest level, domain code is either a country code (for example, uk for the United Kingdom, ie for Ireland, de for Germany, za for South Africa, au for Australia) or, in the United States, denotes a type of organisation (com for commercial organisations, edu for educational establishments, gov for government bodies, mil for the military, org for other organisations, and net for network resources). The subsequent domain codes indicate progressively smaller networks (ac for academic organisations, ic

Received: from judy.any.ac.uk by bccmsa.bc.any.ac.uk (4.1/4.0) id AA12621; Mon, 12 Jun 95 13:41:52 BST

Received: from triumph.doc.any.ac.uk (actually passion.doc.any.ac.uk) by judy. any.ac.uk with SMTP (PP); Mon, 12 Jun 1995 13:41:34 +0100

Message-ld: <m0sL8nz-000DkfC@triumph.doc.any.ac.uk>

Date: Mon, 12 Jun 95 13:41 BST

From: jqs1@doc.any.ac.uk (John Smith)

To:m.pallen@any.ac.uk,f.bloggs@any.ac.uk

Subject: Re: Travel to phage genetics meeting

CC: j.doe@any.ac.uk

Reply-to: j.q.smith@any.ac.uk

Hi Mark! Hi Fred!

$>$ Any idea what time the trains go?

I have just rung $B R$ and the times from London to Manchester are every hour on the hour taking 2:30-so IMHO 11:00 is the best option.

Please let me know if this is not convenient_before_next Tuesday. And ... please can everyone get there *on time*!

$>$ and where do you think we should meet?

Memory and a little map in my diary :-) shows that we travel from Euston, I suggest that we meet there.

Cheers

John

\begin{tabular}{|c|c|}
\hline 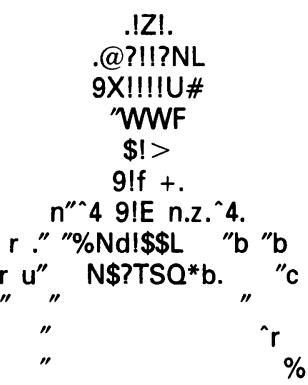 & $\begin{array}{l}\text { Dr John Smith } \\
\text { Phage geneticist } \\
\text { j.q.smith@any.ac.uk } \\
\text { “A bacterium is just a } \\
\text { phage's way of making } \\
\text { more phage..." }\end{array}$ \\
\hline
\end{tabular}

Fig 1-A typical email message for Imperial College, bc for the Department of Biochemistry) until the leftmost domain code, which identifies the machine that runs the email software (the "mail server"). Some institutions have aliasing systems in place so that the email address can be abbreviated, for example, in this case to:

m.pallen@ic.ac.uk

\section{Email conventions}

The body of an email contains the message itself. A standard email message consists of simple text, without the sophistication of bold, underlining, or italics, written in just 128 (in jargon: "7 bit") ASCII characters (the American Standard Code for Information Interchange-a limited set of characters, lacking, for example, the $£$ sign). This apparent limitation, in fact, exerts profound and largely beneficial effects on the social and professional dynamics of communication by email. ${ }^{23}$ Several factors account for this:

- Email messages often carry little information about the social status, hierarchical position, race, age, or appearance of the sender

- Emails lack fancy letterheads or corporate logos

- Email turnaround times are fast-two individuals can exchange several messages a day, leaving little time for formalities.

These factors mean that email messages are usually written in a less formal, more conversational style than conventional letters. They are likely to start with "Hi!" rather than with "Dear Dr Smith" and more likely to end with "Cheers" than "Yours sincerely." This ease of use and informality often make email the preferred medium of communication. You can get so accustomed to using email that you feel disappointed if a new acquaintance or collaborator does not have an email address.

Because email is a text only medium certain rules need to be followed for clear and easy communication (see fig 1). ${ }^{9}$ There should be generous use of white space, with the message divided into many small paragraphs separated by blank lines. When replying to an email message it often helps to quote some of the original message; this may be done automatically by the email software. Quoted lines are usually prefaced by a "> " or a ":". Prolonged use of capital letters is frowned on as the on line equivalent of shouting. Key words are best emphasised by surrounding them with asterixes (for example, ${ }^{\star}$ on time ${ }^{\star}$ ) or underscores (_before_).

Veteran email users make use of a set of electronic substitutes for body language or tone of voice, known as "smileys" or "emoticons." "These are faces made out of ASCII characters. Smileys may seem silly if you are not used to them, but you will often see them in email and elsewhere on the net. To appreciate their meaning you must, mentally at least, turn your head sideways. Here are some of the more common emoticons, with examples of their use:

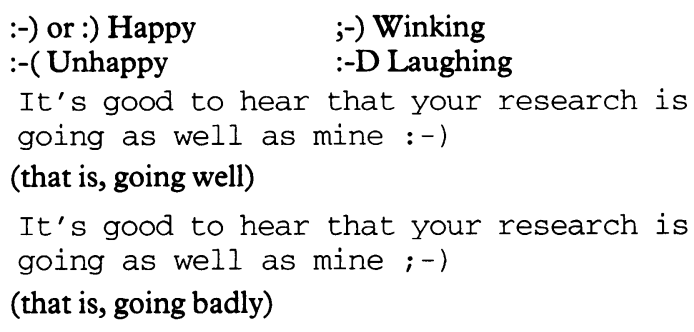

You will often see acronyms in email, such as IM(H)O for "in my (humble) opinion," BTW for "by the way," FYI for "for your information," and TIA for "thanks in advance."

An email often ends with a block of text known as a "sig" or "signature" that is added automatically to all 
outgoing email messages. Your email signature will usually contain your name, address, and phone number; your preferred email address(es); and perhaps some other personal information. Your signature file might also contain a short quotation expressing your view on life and/or some ASCII art-that is, a picture created with ASCII characters (fig 1).

\section{Email client programs}

If you access the Internet through a shell account, you can either use the mail program that is built in to the UNIX environment or try a more user friendly alternative such as PINE or ELM. However, if you have full Internet access, you are better off using an email client program on your own microcomputer, so that you can compose or read messages off line (saving phone bills if you are connecting via a modem) and work within a graphical user interface (such as the Mac OS, OS $/ 2$, or Windows) that is more user friendly than UNIX. These programs also let you set up menus listing the email addresses you commonly use and multiple subject oriented mailboxes, so that your ingoing and outgoing messages can be stored according to subject matter (fig 2). Many email client programs can be downloaded via the Internet and are free to academic users. Eudora ${ }^{11}$ and Pegasus, ${ }^{12}$ two popular email client programs, are available for both Macintosh and Windows operating systems.

\section{Finding people by email: Finger and Netfind}

Even in this electronic age, the quickest way to find out someone's email address is often to phone or write and ask them for it. However, there are several on line methods that can be used to find or check someone's email address. Universities often keep email directories that can be searched via the world wide web ${ }^{13}$ or by telnet. ${ }^{14}$

Other approaches to finding email addresses include Netfind, which is accessible by telnet (log in as Netfind), ${ }^{15}$ gopher, ${ }^{1617}$ and the "Whois" service. ${ }^{18}$ If these are no help, and you know where the person works, you can try sending a request for an email address to the postmaster account on any machine at their workplace (address the message to postmaster@<machinename $>$ ).

If you have an email address and want to know more about its owner, you can use a tool known as Finger. If you have a UNIX account, try typing

finger mjp123@bccmsa.bc.ic.ac.uk

to receive information about me, such as when I last logged in and some personal details that I have made public by placing them in a special "plan" file. If you

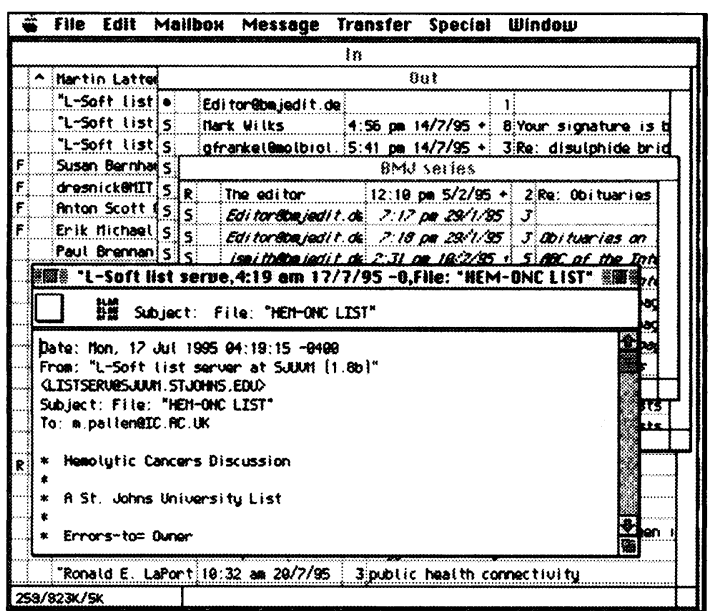

Fig 2-Eudora provides an attractive and intuitive email interface

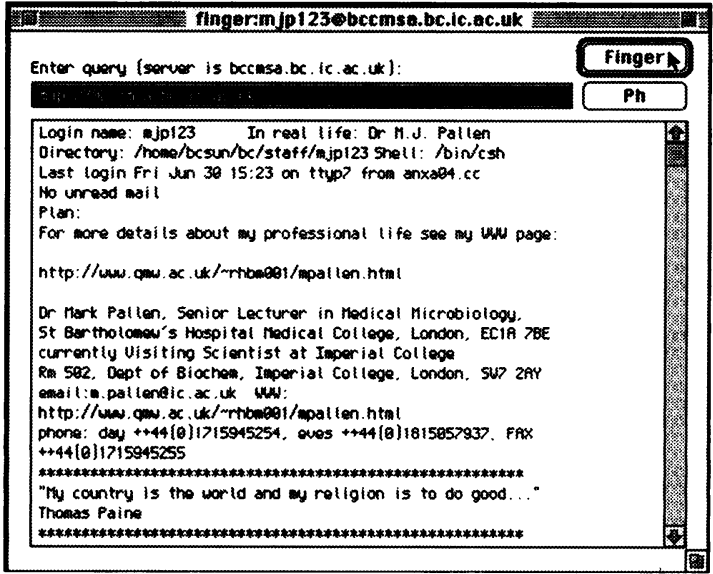

Fig 3-A finger query

have full Internet access, you may find it easier to use Finger through suitable client programs such as Eudora (fig 3) or through the world wide web. ${ }^{19}$ Note that fingering m.pallen@ic.ac.uk will not work; you need to finger the full email address (mjp123@bccmsa. bc.ic.ac.uk).

\section{Advanced uses of email}

FILE ENCODINC

Although email messages are written in ASCII characters, you can use email to send more complex "binary" files, such as programs or pictures, if you encode these files into ASCII characters before sending them. The recipient will have to decode them into the original format on receipt. The earliest system for encoding binary files into ASCII was "uuencode" (for UNIX to UNIX encode), which is built into the UNIX operating system. You can use uuencode on your microcomputer if you have suitable software. BINHEX is another similar encoding system.

In the past, if you wanted to send a big file by email you had to break the encoded file up into small chunks and then send each chunk as the body text of a separate message. The receiver would then have to reassemble the chunks into a single file before decoding. Loss of a single character during chunking or reassembly could spell disaster. Fortunately, many email systems now support a specification known as MIME (multimedia inline media extensions), which allows you to attach a binary file to an email message in such a way that the recipient's email program saves the attached program directly to disk in the correct format.

\section{ELECTRONIC MAILING LISTS}

Electronic mailing lists are a valuable resource that you can exploit by email. In the simplest case, an electronic mailing list can be created manually by putting multiple recipients on the Send-to: line; any of the recipients can then reply to all the others, using the Reply-to-all option available on most email programs.

More sophisticated mailing lists are administered automatically by dedicated programs called listservers. Here, the subscriber sends commands (usually included in the body of the message) to the listserver at one email address and sends contributions for inclusion in the mailing list to another address. Contributions to the list are sent out to all those subscribed to the list (who may number in the thousands). There are several common listserver software packages, including Listserv, Majordomo, Listprocessor, and the British based Mailbase. ${ }^{20}$ The syntax used to send commands to a listserver varies with the type of program. Fortunately all listservers will respond to an email containing the single word "help." 
Selected medical mailing lists

\begin{tabular}{|c|c|c|c|}
\hline Subject & List name & Subscription address & Subscription command \\
\hline Academic dermatology & ACADERM-L & listproc@ucdavis.edu & subscribe acaderm- $L$ firstname lastname \\
\hline Academic family medicine & FAMILY-L & listserv@mizzoul.missouri.edu & Subscribe FAMILY-L \\
\hline Anaesthesiology & ANESTHESIOLOGY & listproc@gasnet.med.nyu.edu & subscribe anesthesiology \\
\hline Cancer-L & CANCER-L & listserv@wvnvm.wvnet.edu & Subscribe CANCER-L \\
\hline Diarrhoeal diseases and disorders & DIARRHOE & listserv@searn.sunet.se & Subscribe DIARRHOE \\
\hline Down's syndrome & DOWN-SYN & listserv@vml.nodak.edu & Subscribe DOWN-SYN \\
\hline Emergency medicine practitioners & EMED-L & majordomo@itsa.ucsf.edu & Subscribe EMED-L \\
\hline Forensics & FORENS-L & listserv@acc.fau.edu & Subscribe FORENS-L \\
\hline Geriatric health care & GERINET & listserv@ubvm.cc.buffalo.edu & Subscribe GERINET \\
\hline Inflammatory bowel diseases & IBDLIST & ibdlist-request\%mvac23@udel.edu & personal request \\
\hline Internet health resources & HMATRIX-L & listserv@kumchttp.mc.ukans.edu & SUBSCRIBE HMATRIX-L YOUR_NAME \\
\hline Leukaemia, lymphoma, myeloma & HEM-ONC & listserv@sjuvm.stjohns.edu & subscribe hem-onc First-Name Last-Name \\
\hline Medical imaging & MEDIMAGE & listserv@vm.poly.edu & Subscribe MEDIMAGE \\
\hline Medical Informatics & MMATRIX-L & listserv@kumchttp.mc.ukans.edu & SUBSCRIBE MMATRIX-L YOUR_NAME \\
\hline Neurologists Forum & NEURO & neuro@emgmhs.mcg.edu & Subscribe Neuro \\
\hline Neurosurgery & NEUROSURGERY & listserv@gibbs.oit.unc.edu & subscribe neurosurgery firstname lastname \\
\hline Obstetrics and gynaecology & OB-GYN-L & listserv@bcm.tmc.edu & subscribe ob-gyn-I yourfirstname your lastname \\
\hline Paediatric intensive care unit & PICU & listproc@its.mcw.edu & subscribe picu Firstname Lastname \\
\hline Radiology & RADSIG & listserv@uwavm.u.washington.edu & Subscribe RADSIG \\
\hline Rheumatology and arthritis & ARTHRITIS-L & listproc@showme.missouri.edu & subscribe arthritis-I \\
\hline Schizophrenia information exchange & SCHIZOPH & listserv@vm.utcc.utoronto.ca & Subscribe SCHIZOPH \\
\hline Stroke & STROKE-L & listserv@ukcc.uky.edu & Subscribe STROKE-L \\
\hline Women's health & WMN-HLTH & listserv@uwavm.u.washington.edu & Subscribe WMN-HLTH \\
\hline
\end{tabular}

As a new subscriber to a mailing list you will receive an automatic acknowledgement of the subscription, together with instructions on how to contribute to and, most importantly, how to unsubscribe from the mailing list. This latter point is particularly important as some mailing lists can soon fill up your mailbox (particularly if you go on holiday for a few weeks). Take care at this point to examine the ground rules for the mailing list. For example, the mailing list may be restricted to medical specialists or may be intended also for patients.

There are hundreds of electronic mailing lists of relevance to medicine and the biomedical sciences (table). Information on such lists can be obtained from the medical matrix ${ }^{21}$ and other sources. ${ }^{22-24}$ As most medical electronic mailing lists have a decidedly North American bias, British readers may be particularly interested in joining or even founding biomedical mailing lists within the Mailbase service. Because of the way Mailbase is funded, all Mailbase lists must primarily be of benefit to the higher education and research community in Britain. Some of the more popular Mailbase lists include those in the box. To subscribe to any of these, send the message

$$
\begin{aligned}
& \text { JOIN <listname> } \\
& \text { <yourfirstname }(\mathrm{s})><\text { yourlastname> }
\end{aligned}
$$

(where $<$ listname $>$ is one of the above list names) as the sole text of an email to mailbase@mailbase.ac.uk

evidence-based-health medical-it science-education downs-research gp-uk public-health

As noted above, many computers that lack access to core Internet services can still exchange email with machines on the Internet. For this reason, email is often pressed into service for non-mail-like functions. Many of the Internet resources covered later in this clumsily. To learn more about how to use email in this fashion, retrieve Bob Rankin's comprehensive Guide to Offline Internet Access ${ }^{25}$ by sending an email to mailbase@mailbase.ac.uk with this line in the body of the message:

send lis-iis e-access-inet.txt

1 Sands DZ, Safran C, Slack WV, Bleich HL. Use of electronic mail in a teaching hospital. Proc Annu Symp Comput Appl Med Care 1993;17:306-10.

2 Singarella T, Baxter J, Sandefur RR, Emery CC. The effects of electronic mail on communication in two health sciences institutions. $₹$ Med Syst 1993;17: 69-86.

3 Sproull L, Kiesler S. Computers, networks and work. Scientific American 1995; 6 (special issue): 128-39.

4 Lyness AL, Raimond JA. Electronic communication to promote consensusbuilding skills: an innovative teaching strategy. $₹$ Nurs Educ 1992;31:331-4

5 Staggers N. Electronic mail basics. I Nurs Adm 1989;19:31-5.

6 Fridsma DB, Ford P, Altman R. A survey of patient access to electronic mail: attitudes, barriers, and opportunities. Proc Annu Symp Comput Appl Med Care 1994;18:15-9.

7 Neill RA, Mainous AR, Clark JR, Hagen MD. The utility of electronic mail as a medium for patient-physician communication. Arch Fam Med 1994;3 268-71.

8 Letterie GS, Morgenstern LL, Johnson L. The role of an electronic mail system in the educational strategies of a residency in obstetrics and gynecology. Obstet Gynecol 1994;84:137-9.

9 Angell D, Heslop B. The elements of e-mail style. Reading, MA: AddisonWesley, 1994.

10 Sanderson DW. Smileys. Sebastopol, CA: O'Reilly \& Associates, 1994.

11 QUEST home page: http://www.qualcomm.com/quest/QuestMain.html

12 Pegasus Mail Information: http://mirkwood.ucc.uconn.edu/pmail.htm

13 Query X500 Directory Services Database: http://www.ic.ac.uk/ccs/nss/ Directory-Services/X500Query.html

14 Paradise X.500 Interface: telnet://paradise.ulcc.ac.uk

15 Netfind by telnet: telnet://monolith.cc.ic.ac.uk

16 Netfind by gopher: gopher://ds.internic.net:4320/7netfind\%20dblookup

17 Netfind by world wide web: http://info.lut.ac.uk/dir/netfind.html

18 Guide to network resource tools: http://www.earn.net/gnrt/whois.html

19 InterLink Finger gateway: http://henry.interlink.no/finger

19 InterLink Finger gateway: http://henry.interlink.no/fing

20 Mailbase mailing list service: http://mailbase.ac.uk/ Medical matrix guide to internet medical resources: series (file transfer protocols, gopher, the world wide web) are accessible through email, albeit often
22 Publicly accessible mailing lists: http://www.neosoft.com/internet/paml

23 Kovacs 9th revision directory of scholarly electronic conferences: medical practice/nursing/medical personnel/patients: gopher://nisp.ncl.ac.uk:70/00 /other/kovacs/acadlist.medical

24 Kovacs 9th revision directory of scholarly electronic conferences: gopher://

25 Accessing the internet by e-mail: Doctor Bob's guide to offline Intermet access, 4th ed, July 1995: ftp://mailbase.ac.uk/pub/ists/is-iis/files/e-access-inet.txt nisp.ncl.ac.uk:70/00/other/kovacs/ 